OPEN ACCESS

Edited by:

Oswaldo Valdes-Lopez, Universidad Nacional Autónoma de

México, Mexico

Reviewed by:

Norberto Daniel lusem,

Universidad de Buenos Aires,

Argentina

Jose Luis Reyes,

Universidad Nacional Autónoma de

México, Mexico

${ }^{*}$ Correspondence:

Venu Kalavacharla

vkalavacharla@desu.edu

Specialty section:

This article was submitted to

Plant Nutrition,

a section of the journal

Frontiers in Plant Science

Received: 29 October 2015

Accepted: 22 March 2016

Published: 26 April 2016

Citation:

Crampton M, Sripathi VR, Hossain K and Kalavacharla V (2016) Analyses of Methylomes Derived from

Meso-American Common Bean (Phaseolus vulgaris L.) Using MeDIP-Seq and Whole Genome Sodium Bisulfite-Sequencing.

Front. Plant Sci. 7:447. doi: 10.3389/fpls.2016.00447

\section{Analyses of Methylomes Derived from Meso-American Common Bean (Phaseolus vulgaris L.) Using MeDIP-Seq and Whole Genome Sodium Bisulfite-Sequencing}

\author{
Mollee Crampton ${ }^{1}$, Venkateswara R. Sripathi ${ }^{1}$, Khwaja Hossain ${ }^{2}$ and \\ Venu Kalavacharla ${ }^{1,3 *}$
}

${ }^{1}$ Molecular Genetics and Epigenomics Laboratory, Delaware State University, Dover, DE, USA, ${ }^{2}$ Division of Science and Mathematics, Mayville State University, Mayville, ND, USA, ${ }^{3}$ Center for Integrated Biological and Environmental Research, Delaware State University, Dover, DE, USA

Common bean (Phaseolus vulgaris L.) is economically important for its high protein, fiber, and micronutrient contents, with a relatively small genome size of $\sim 587 \mathrm{Mb}$. Common bean is genetically diverse with two major gene pools, Meso-American and Andean. The phenotypic variability within common bean is partly attributed to the genetic diversity and epigenetic changes that are largely influenced by environmental factors. It is well established that an important epigenetic regulator of gene expression is DNA methylation. Here, we present results generated from two high-throughput sequencing technologies, methylated DNA immunoprecipitation-sequencing (MeDIP-seq) and whole genome bisulfite-sequencing (BS-Seq). Our analyses revealed that this Meso-American common bean displays similar methylation patterns as other previously published plant methylomes, with $\mathrm{CG} \sim 50 \%, \mathrm{CHG} \sim 30 \%$, and $\mathrm{CHH} \sim 2.7 \%$ methylation, however, these differ from the common bean reference methylome of Andean origin. We identified higher $\mathrm{CG}$ methylation levels in both promoter and genic regions than $\mathrm{CHG}$ and $\mathrm{CHH}$ contexts. Moreover, we found relatively higher CG methylation levels in genes than in promoters. Conversely, the $\mathrm{CHG}$ and $\mathrm{CHH}$ methylation levels were highest in promoters than in genes. This is the first genome-wide DNA methylation profiling study in a MesoAmerican common bean cultivar ("Sierra") using NGS approaches. Our long-term goal is to generate genome-wide epigenomic maps in common bean focusing on chromatin accessibility, histone modifications, and DNA methylation.

Keywords: Phaseolus vulgaris, common bean, methylome, whole genome bisulfite-sequencing, MeDIP-Seq

\section{INTRODUCTION}

Common bean (Phaseolus vulgaris) is the most widely consumed legume, as it is high in protein, fiber, and essential nutrients, while low in glycemic index (Mitchell et al., 2009). The haploid genome size of common bean is about $587 \mathrm{Mb}$ (Schmutz et al., 2014). Bean domestication is assumed to be a complex process that involved two distinct gene pools (Meso-American and Andean) and repeated selection of desirable traits within the gene pool (Bitocchi and Nanni, 2012). 
Common bean is consumed widely across the world due to its affordability and health benefits (Gepts et al., 2008). Food legumes such as common bean are an inherently rich source of vitamins and minerals, including $B$ vitamins (particularly B1/thiamine and B9/folic acid), iron, zinc, calcium and magnesium (Akond et al., 2011; Beebe et al., 2013; Câmara et al., 2013; Petry et al., 2015). Hence, common bean is considered as a viable target for biofortification (Blair, 2013). In order to effectively modulate genetic architecture and develop micronutrient-enriched crops, understanding the genes and their regulatory mechanisms involved in physiological processes is important. Though several regulatory mechanisms have been identified, DNA methylation, chromatin remodeling, histone acetylation, histone methylation, and gene silencing are of paramount importance.

DNA methylation is a covalent, heritable epigenetic modification that plays a significant role in gene expression, tissue specialization, and transposon inactivation (Zhang et al., 2006; Verhoeven et al., 2010). In plants, cytosine methylation occurs in both symmetrical (CG and $\mathrm{CHG}$ ) and asymmetrical $(\mathrm{CHH})$ contexts, while descending order of the extent of methylation in these contexts is $\mathrm{CG}, \mathrm{CHG}$, and $\mathrm{CHH}$, where $\mathrm{H}$ indicates A, C, or T (Lister and Ecker, 2009; Henderson et al., 2010; Saze et al., 2012). DNA methylation occurs at higher levels in heterochromatin when compared to euchromatin (Madlung and Comai, 2004; Song et al., 2013). Genome-wide DNA methylation and gene expression studies revealed that gene-body methylation is conserved across species (Feng et al., 2010), among constitutively expressed genes (Zhang et al., 2006), and more specifically, CG methylation is often linked to increased gene expression (Miura et al., 2009; Wang et al., 2014; Kim et al., 2015). Conversely, promoter DNA methylation in plants is largely associated with transcriptional repression (Li et al., 2008) and is highly tissue-specific in nature (Zhang et al., 2006; Song et al., 2013). In soybean (dicot) and rice (monocot), it has been reported that promoter hypomethylation resulted in increased gene expression of flanking genes ( $\mathrm{Li}$ et al., 2008; Song et al., 2013).

There are several methods for determining the presence and location of methylated cytosines in the genome, which include methylation-sensitive restriction enzyme sequencing (MRE-seq), affinity purification, and whole genome bisulfite-sequencing (BS-Seq). This study used one of the three affinity purification methods, i.e., methylated DNA immunoprecipitation sequencing (MeDIP-seq) and BS-Seq. MeDIP-Seq utilizes anti-5-mC antibody and generates $\sim 10^{7}$ reads while BS-Seq being a "gold standard" of methylation sequencing, generates $\sim 10^{8}$ methylated cytosines at single nucleotide resolution. This technique converts unmethylated cytosines to uracil by deamination, while methylated cytosines remain as cytosines (Lister and Ecker, 2009; Laird, 2010; Kim et al., 2014). A genome-wide DNA methylation map has been reported in Andean common bean (Kim et al., 2015) but it is not available in Meso-American common bean. The previous methylome study compared the methylation in Andean common bean leaf to soybean leaf, stripped root, and root hair methylomes (Kim et al., 2015). Screening the methylomes of Meso-American common bean (P. vulgaris) using highthroughput approaches such as BS-Seq and MeDIP-Seq for genome-wide DNA methylation may aid in understanding key regulatory mechanisms linked to important biological processes.

\section{RESULTS}

Whole genome bisulfite-sequencing (BS-Seq) is widely used to study DNA methylation at the whole genome level while MeDIP-Seq is used to detect high DNA methylation in lowdensity $\mathrm{CG} / \mathrm{CHG} / \mathrm{CHH}$ areas or moderate methylation levels in high-density $\mathrm{CG} / \mathrm{CHG} / \mathrm{CHH}$ regions. To understand the DNA methylation levels in the three contexts (CG, CHG, and $\mathrm{CHH}$ ), we constructed two MeDIP-Seq libraries (one input control that served as no antibody sample and one immunoprecipitated sample) and one BS-Seq library using tissue collected from three sets of bean plants from the Meso-American common bean cultivar, Sierra, grown at three different times (three biological replicates grown in triplicate) which were pooled and sequenced on the Illumina/HiSeq2500 platform. A similar replicate pooling approach has been used in other epigenomic studies that utilized next generation sequencing technologies (Taylor et al., 2007; Brinkman et al., 2012; Statham et al., 2012). The libraries generated in this study were labeled as Sierra_Input, Sierra_MeDIP and Sierra_BS (Table 1). The input control (Sierra_Input) was used for comparing against immunoprecipitated DNA (Sierra_MeDIP) during MeDIP-Seq analysis to identify differentially methylated regions (DMRs).

\section{Data Collection and Preprocessing}

Deep sequencing of three methylome libraries in common bean resulted in $\sim 260$ million-50 bp Illumina reads (Table 1). Of these, $\sim 214$ million reads were from BS-Seq and the rest ( $\sim 77$ million reads) were from MeDIP-Seq. Among MeDIPSeq reads, $\sim 22.5$ million reads were from input control and $\sim 23.7$ million were methylated DNA immunoprecipitated reads. The raw reads obtained from both methodologies were trimmed, filtered, and high quality reads collected were aligned to the reference $P$. vulgaris genome, G19833 (Schmutz et al., 2014) available at Phytozome (V1.0, accessed October 2014). In total, $661.4(28.67 \%), \sim 8.9(39.53 \%)$ and 7.2 (30.25\%) million BS-Seq, MeDIP-Seq input control, and MeDIP-Seq immunoprecipitated reads were mapped to the reference genome (G19833). Of these, uniquely mapped reads with $\leq 2$ mis-matches were further used in analysis.

\section{Bisulfite-Sequencing (BS-Seq)}

DNA methylation ratios from BS-Seq were evaluated for CG, $\mathrm{CHG}$, and $\mathrm{CHH}$ contexts. The increasing order of methylation levels in the three contexts were $\mathrm{CHH}$ followed by $\mathrm{CHG}$ and CG. Though the methylation patterns for $\mathrm{CG}$ and $\mathrm{CHG}$ contexts were similar, the overall levels of $\mathrm{CHG}$ methylation were 
TABLE 1 | Reads and mapping ratios for each sample.

\begin{tabular}{|c|c|c|c|c|}
\hline Sample name & Method & Total read \# & Mapped read \# & Mapping ratio (\%) \\
\hline Sierra_BS & BS-Seq & $214,016,819$ & $61,362,998$ & 28.67 \\
\hline Sierra_Input & INPUT & $22,509,873$ & $8,897,764$ & 39.53 \\
\hline Sierra_MeDIP & MeDIP-seq & $23,709,423$ & $7,173,104$ & 30.25 \\
\hline
\end{tabular}

relatively lower than CG methylation. The genome-wide DNA methylation levels for each context are shown (Figures 1A,B). The least methylated context, $\mathrm{CHH}$ was consistently very low across all 11 common bean chromosomes. In addition to the overall methylation across the three contexts, we also investigated the methylated cytosines in promoter and genic regions. The promoter and gene coverage between CG (67\%, 71\%) and CHG $(71 \%, 73 \%)$ contexts varied when compared to $\mathrm{CHH}$ coverage (95\%, 90\%) context. While the majority of the reads covered in all contexts were more than 50 -fold at each cytosine site, some sites were not covered (Figure 2). For each context, the overall read coverage (number of reads per cytosine) was determined as either 0 (no coverage), 1-4, 5-10,11-20, and $>20$ reads per cytosine (Supplementary Figure S2). The majority of the covered reads was more than 11 reads per cytosine and this trend was consistent across the three contexts of methylation measured by BS-Seq.

Methylation ratios and their frequencies throughout the genome were analyzed in each context. Within the CG context, $\sim 2.65$ million total sites and $\sim 1.25$ million methylated sites $\geq 0.8$ ( $\sim 50 \%)$ were identified across the genome. In the CHG context, $\sim 3.3$ million total sites and $\sim$ one million methylated sites ( $\sim 30 \%$ ) were identified. The most prominent context was $\mathrm{CHH}$ and we identified approximately 18 million $\mathrm{CHH}$ sites in the genome, but only $\sim 500,000$ sites were methylated $(2.7 \%)$ with the vast majority of sites unmethylated. Our results show that most of the methylation and highly methylated regions are found outside of the promoter and genic regions (intragenic) in all three contexts (Figure 3A). Methylation levels for the annotated promoter and genic regions are presented in box plots for each context (Figure 3B). Higher CG methylation was found in genic regions when compared to the promoters. Conversely, comparatively higher $\mathrm{CHG}$ and $\mathrm{CHH}$ methylation was observed in promoter regions than genic regions. However, the overall CG methylation was highest among the three contexts.

\section{Methylated DNA Immunoprecipitation (MeDIP-Seq)}

To understand the methylome of common bean, assessing the peak density and peak shape associated with DNA methylation is necessary. Peaks can be called by mapping the reads
A
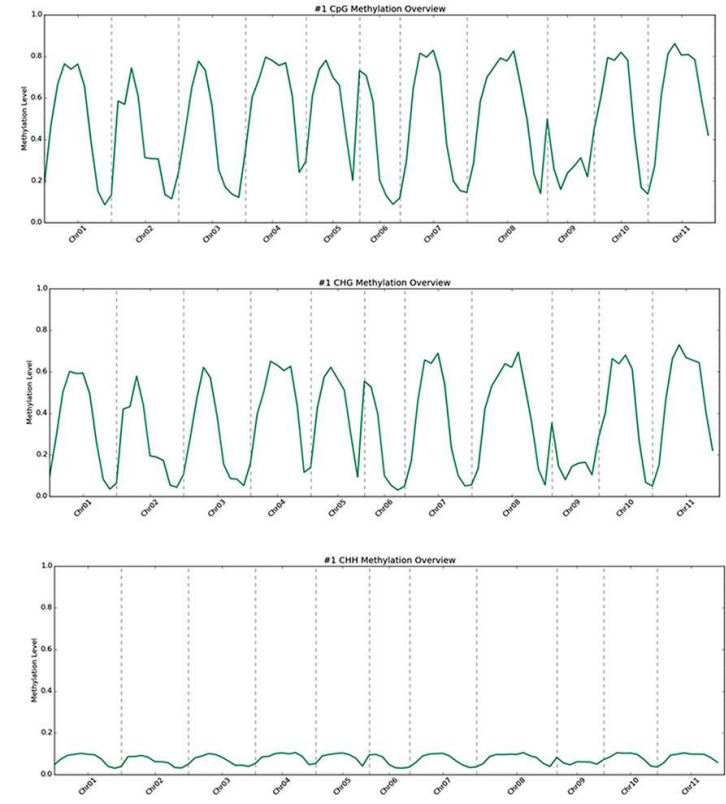

B
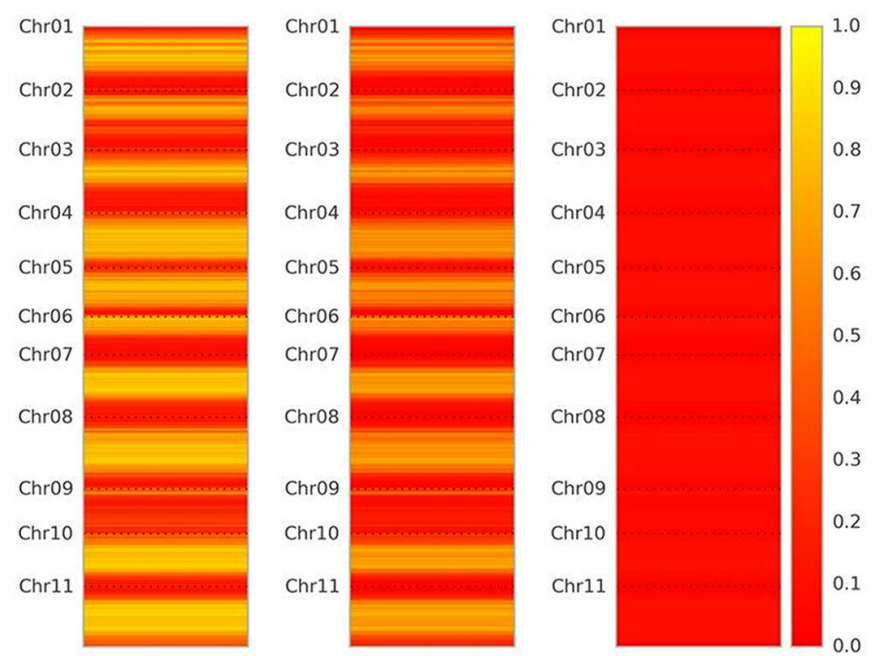

FIGURE 1 | (A) Whole genome overview of DNA methylation levels, by context. The top is CG methylation, middle is $\mathrm{CHG}$ methylation, and bottom is CHH methylation levels across all 11 chromosomes of common bean. The scale ranges from 0.0 (no methylation) up to 1.0 (100\% methylation). (B) Heat map representation of methylation levels, by context. The left is CG methylation, the middle is $\mathrm{CHG}$ methylation, and the right is $\mathrm{CHH}$ methylation levels across all 11 chromosomes of common bean. The scale ranges from 0.0/red (no methylation) up to 1.0/yellow (100\% methylation). 

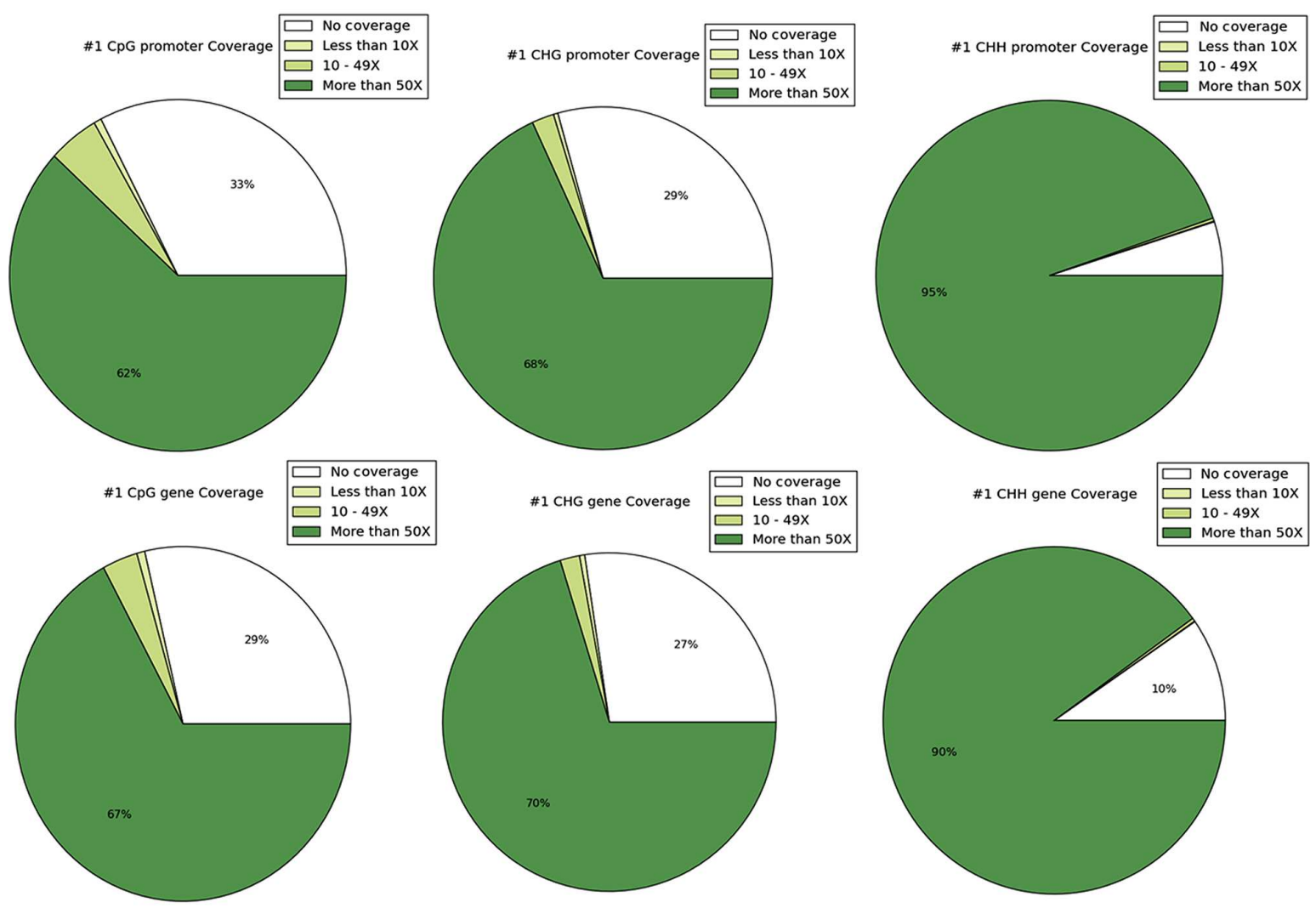

FIGURE 2 | The coverage of sites is shown and depicted in the categories of 0 coverage, $<10 \times$ coverage, 10-49x coverage, and $\geq 50$ coverage. The top row shows coverage by context in promoters and the bottom row shows coverage by context in genes.

to the genome to reveal the loci of selectively methylated DNA. Several peak callers are currently available to identify the global pattern of these modified sites by comparing the antibody tagged sample with its background (input, no antibody). The shapes are based on genomic coverage of the input control, the coverage of the DNA that was not subjugated to immunoprecipitation with an anti-5mC antibody, versus the immunoprecipitated DNA. The increased coverage is displayed as a "peak," which implies that there is more coverage, due to the enrichment of DNA containing methylated cytosines (Supplementary Figure S1). Peak assessment is very important in minimizing the false discovery rate and developing a goldstandard common bean methylome. A correlation between peak density and chromosomal length has been proposed in mammals (Hughes et al., 2010). We estimated the methylation peak density (peak/Mb) based on chromosome length and total number of peaks identified per chromosome. MeDIP-Seq signatures per chromosome, total peaks across the genome, peaks found only in promoter regions, and peaks identified in genic locations are presented (Table 2). The majority (93.2\%) of the peaks identified from MeDIP-Seq analysis were in non-promoter and non-genic regions. The peaks obtained from MeDIP-Seq were compared against the methylation sites from BS-Seq data to corroborate the peaks identified in likely methylated regions and to compare the two methylome sequencing work flows. The top
20 most significant $(p<0.05)$ MeDIP-Seq peaks were compared to $\mathrm{CG}$ and $\mathrm{CHG}$ weighted methylation determined from BSSeq to identify that all of these methylated cytosine regions possess methylation ratios over 0.5 in CG and/or CHG contexts (Table 3).

\section{DISCUSSION}

DNA methylation is a heritable epigenetic mark that controls a wide variety of physiological processes (Boyko et al., 2007). Though DNA methylation has been reported in many eukaryotic organisms, the contexts and levels of DNA methylation vary significantly between plants and animals. In animals, the symmetric context, CG, is most prominent while non-CG (CHG and $\mathrm{CHH}$ ) methylation is less frequent (Stroud et al., 2013b). In plants, DNA is primarily methylated in the CG context while non-CG contexts $(\mathrm{CHG}$ and $\mathrm{CHH}$ ) are also abundant. The reports on CG and non-CG methylation in plants are increasingly evident. For example, in Arabidopsis both CG and non-CG methylation are functional (Law and Jacobsen, 2010) and the roles of chromomethyltransferase (CMT) and domains rearranged methyltransferase (DRM) proteins in nonCG methylation has been established (Cao et al., 2003; Stroud et al., 2013b). 


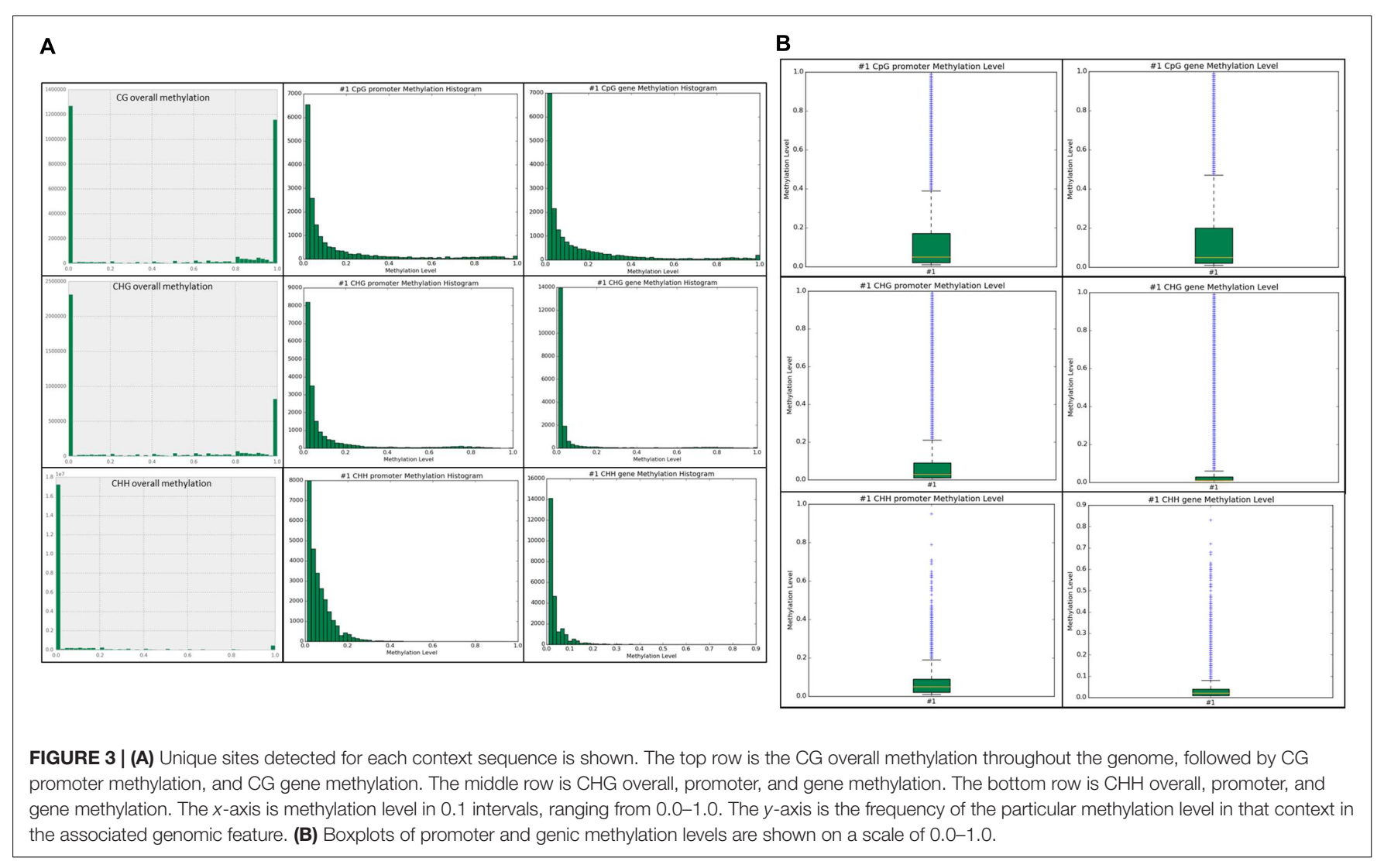

TABLE 2 | Common bean chromosomal length, reads, signatures, and total and annotated peaks from MeDIP-Seq data.

\begin{tabular}{|c|c|c|c|c|c|c|}
\hline Reference & Chrom length (Mbp) & Reads & Signatures & Total peaks & Peaks in promoter & Peaks in genes \\
\hline ChrO2 & 49 & $1,413,718$ & 27,421 & 2,977 & 103 & 99 \\
\hline ChrO4 & 45.9 & $1,916,912$ & 31,458 & 2,943 & 92 & 65 \\
\hline Chr05 & 40.8 & $1,548,871$ & 26,404 & 2,572 & 109 & 82 \\
\hline Chr06 & 32 & 967,065 & 17,434 & 1,544 & 81 & 72 \\
\hline Chr08 & 59.7 & $2,877,212$ & 34,359 & 3,774 & 137 & 109 \\
\hline Chr09 & 37.5 & $1,194,008$ & 16,610 & 1,919 & 97 & 71 \\
\hline Chr10 & 43.3 & $1,986,809$ & 29,905 & 3,091 & 105 & 101 \\
\hline Chr11 & 50.4 & $2,473,345$ & 34,568 & 3,740 & 153 & 104 \\
\hline Total & 514.9 & $20,045,287$ & 319,682 & 32,456 & 1,265 & 943 \\
\hline
\end{tabular}

MeDIP-Seq yields a resolution of $\sim 100-200$ bp while single base resolution methylome maps can be obtained by both conventional Sanger sequencing of short BS-treated fragments and whole genome bisulfite-sequencing (Taiwo et al., 2012; Stevens et al., 2013). In common bean, we generated 10 -fold more reads using BS-Seq when compared to MeDIP-Seq, as previously reported (Laird, 2010). The relatively lower number of reads in MeDIP-Seq compared to BS-Seq is due to the inherent difference between the techniques (Laird, 2010). However, MeDIP-Seq analysis identified densely methylated regions in the genome while
BS-Seq analysis estimated overall methylation rates in the genome.

Currently, few reports of BS-Seq are available in plants, which are mostly model species and some are presented (Table 4). Epigenome reports in legume crops are scantly available. Recently, single nucleotide resolution reference methylomes in soybean and Andean common bean have been constructed using BS-Seq in order to understand epigenetic variation in legume crops (Kim et al., 2015). Here we generated single nucleotide resolution (BS-Seq) and $150 \mathrm{bp}$ resolution (MeDIP-Seq) maps of DNA methylation to understand 
TABLE 3 | The top 20 most significant/highest peak shape scores from the MeDIP-Seq data are shown.

\begin{tabular}{|c|c|c|c|c|c|c|c|c|}
\hline Length & Peak shape score & $P$-value & $5^{\prime}$ gene & $5^{\prime}$ distance & $3^{\prime}$ gene & $3^{\prime}$ distance & CG & CHG \\
\hline 243 & 15.03 & $2.30 \mathrm{E}-51$ & & & Phvul.008G000100 & 0 & 0.897252 & N/A \\
\hline 237 & 14.09 & $2.24 \mathrm{E}-45$ & & & Phvul.008G000100 & 95707 & 0.932642 & 0.892308 \\
\hline 231 & 13.66 & $8.72 \mathrm{E}-43$ & & & Phvul.008G000100 & 112068 & 0.686242 & 0.679463 \\
\hline 204 & 13.38 & $3.70 \mathrm{E}-41$ & Phvul.004G046900 & 2257 & Phvul.004G047000 & 4936 & 0.949129 & $\mathrm{~N} / \mathrm{A}$ \\
\hline 210 & 13.29 & $1.35 \mathrm{E}-40$ & Phvul.008G293200 & 8476 & & & 0.850638 & N/A \\
\hline 246 & 12.87 & $3.41 \mathrm{E}-38$ & & & Phvul.008G000100 & 100985 & 0.857233 & 0.726482 \\
\hline 206 & 12.76 & $1.29 \mathrm{E}-37$ & Phvul.008G293200 & 4372 & & & 0.763093 & 0.166279 \\
\hline 226 & 12.71 & $2.57 \mathrm{E}-37$ & Phvul.009G144700 & 60451 & Phvul.009G144800 & 28262 & 0.897214 & 0.823301 \\
\hline 242 & 12.18 & $2.05 \mathrm{E}-34$ & Phvul.008G293200 & 6471 & & & 0.9447 & 1 \\
\hline 209 & 12.03 & $1.23 \mathrm{E}-33$ & Phvul.008G293200 & 8724 & & & 0.92825 & $\mathrm{~N} / \mathrm{A}$ \\
\hline 235 & 11.95 & 3.34E-33 & & & Phvul.008G000100 & 108787 & 0.842105 & 0.88024 \\
\hline 212 & 11.91 & $5.27 \mathrm{E}-33$ & & & Phvul.008G000100 & 113332 & 0.807415 & 0.734861 \\
\hline 221 & 11.43 & $1.41 \mathrm{E}-30$ & Phvul.009G144700 & 50026 & Phvul.009G144800 & 38692 & $\mathrm{~N} / \mathrm{A}$ & 0.775591 \\
\hline 210 & 11.36 & 3.29E-30 & & & Phvul.008G000100 & 110552 & 0.925373 & 0.892 \\
\hline 214 & 11.31 & $5.68 \mathrm{E}-30$ & & & Phvul.008G000100 & 105211 & 0.832864 & 0.814815 \\
\hline 220 & 11.27 & 8.84E-30 & Phvul.009G144700 & 51399 & Phvul.009G144800 & 37320 & 0.5 & 0.779538 \\
\hline 204 & 11.01 & 1.69E-28 & & & Phvul.008G000100 & 109425 & $\mathrm{~N} / \mathrm{A}$ & 0.892105 \\
\hline 206 & 10.83 & $1.26 \mathrm{E}-27$ & Phvul.009G144700 & 58862 & Phvul.009G144800 & 29871 & 0.839901 & 0.689939 \\
\hline 274 & 10.76 & $2.59 \mathrm{E}-27$ & Phvul.009G144700 & 53308 & Phvul.009G144800 & 35357 & 0.850394 & 0.730228 \\
\hline 223 & 10.35 & $2.11 \mathrm{E}-25$ & Phvul.008G293200 & 2479 & & & 0.961965 & N/A \\
\hline
\end{tabular}

Also shown are peak locations in relation to $5^{\prime}$ and $3^{\prime}$ genes. CG and CHG methylation ratios were determined from BS-Seq data in the corresponding peak locations.

\begin{tabular}{ll}
\hline TABLE 4 | Summary of BS-Seq studies in plants. \\
\hline Species & \multicolumn{1}{c}{ Reference } \\
\hline Amborella trichopoda & Albert et al., 2013 \\
Arabidopsis lyrata & Seymour et al., 2014 \\
Arabidopsis thaliana & Cokus et al., 2008; Hseih et al., 2009; Lister \\
& et al., 2009; Greaves et al., 2012; Shen et al., \\
& 2012; Schmitz et al., 2013 \\
Brachypodium distachyon & Takuno and Gaut, 2012 \\
Capsella rubella & Seymour et al., 2014 \\
Glycine max & Schmitz et al., 2013; Song et al., 2013; Kim \\
& et al., 2015 \\
Oryza sativa & Zemach et al., 2010; Chodavarapu et al., 2012; \\
& Li et al., 2012; Stroud et al., 2013a \\
Phaseolus vulgaris & Kim et al., 2015 \\
Solanum lycopersicum & Stroud et al., 2013b \\
Zea mays & Eichten et al., 2013; Gent et al., 2013; Regulski \\
& et al., 2013; West et al., 2014 \\
\hline
\end{tabular}

genome-wide DNA methylation in Meso-American common bean. Further, we focused our analyses in estimating the overall methylation levels, context-specific methylation patterns, and promoter versus gene body methylation patterns. This study represents the first genome-wide DNA methylation report in the important rust-resistant Meso-American common bean cultivar, Sierra.

\section{Genome-Wide Methylation Levels}

We aligned the sequenced cytosines collected from MeDIPSeq and BS-Seq to the common bean reference genome (G19833) to identify genome-wide methylation, site-specific methylation and annotated the methylated peaks. In total, 3.0-3.5 million cytosines were found to be methylated across the genome covering all three contexts of DNA methylation, thus accounting for $<1 \%$ of the total DNA bases in the genome. A total number of 32,456 significant (>1.5-fold-enrichment) peaks were identified in common bean. The average DNA methylation peak density identified was $\sim 63$ peaks/1 Mb of DNA. Based on the average peak density, lower methylation levels were identified in chromosomes, Chr02, Chr03, Chr06, and Chr09. Conversely, higher methylation levels were noted in chromosomes, Chr07, Chr10, and Chr11 (Table 2). Similar patterns in methylation saturation between chromosomes were highlighted by using chromosome-based methylation distribution heat maps (Figure 1B). Genome-wide methylation identified in this study was over $37.8 \%$ in CG and CHG contexts, which is in accordance with the other reports in Arabidopsis, rice and soybean (Cokus et al., 2008; Li et al., 2012; Song et al., 2013).

\section{Context-Specific Methylation Patterns}

In common bean, we generated methylation histograms in order to show the methylation ratios $(0.0-1.0)$ for the cytosine sites in each context within the genome and for the annotated genomic features (Figure 2). The majority of the overall methylation ratios for hemi-methylated or fully methylated sites ranged between 0.8 and 1.0 in the CG context, which is in accordance with CG methylation in Arabidopsis. The distribution of methylation ratios in the $\mathrm{CHG}$ context for the hemi-methylated or fully methylated sites ranged between mainly 0.6-1.0 while in Arabidopsis the ratios reported were between 0.2 and1.0 (Cokus et al., 2008). Within bean, the 
CG and CHG distributions were similar except for fewer fully methylated sites (ratio $=1.0$ ) in the CHG context. The $\mathrm{CHH}$ methylation ratios were predominately $<10 \%$ in Arabidopsis (Cokus et al., 2008; Feng et al., 2010), maize (Eichten et al., 2013), and many other eukaryotic organisms (Feng et al., 2010) which is consistent with the present findings in common bean. As $\mathrm{CHH}$ methylation is commonly found in repetitive and transposable elements, it varies significantly more between tissues and even among biological replicates than CG and CHG methylation (Song et al., 2013; Kim et al., 2015).

In addition to the methylation ratios, the overall methylation levels in our study estimated for $\mathrm{CG}, \mathrm{CHG}$ and $\mathrm{CHH}$ contexts were $\sim 50, \sim 30$, and $\sim 2.7 \%$, respectively. In Arabidopsis, the methylation levels specific to each context ( $~ 24 \%$ in CG, $6.7 \%$ in $\mathrm{CHG}$, and $1.7 \%$ in $\mathrm{CHH}$ contexts) were reported (Cokus et al., 2008). A similar study which screened eight eukaryotic species identified $\sim 22 \%$ of CGs, $\sim 6.0 \%$ of CHGs, and $\sim 2 \%$ of CHHs as methylated in Arabidopsis (Feng et al., 2010). In poplar, the methylation level, $41.9 \%, 20.9 \%$, and $3.25 \%$ were reported for $\mathrm{CG}, \mathrm{CHG}$, and $\mathrm{CHH}$ contexts, respectively (Feng et al., 2010). Recently, among legumes, the methylation percentage for CG (74\% and 64\%), CHG (62\% and $48 \%)$ and $\mathrm{CHH}(21 \%$ and $4 \%)$ contexts have been reported in common bean and soybean leaves, respectively (Kim et al., 2015). Other leaf methylation levels reported in soybean include, $63 \%$ in CG, $44 \%$ in $\mathrm{CHG}$, and $5.9 \%$ in $\mathrm{CHH}$ contexts (Song et al., 2013). Our results were more similar to the methylation levels for each context in poplar than in soybean. This is likely attributed to the fact that bean and poplar have a similar genome size when compared to soybean. Moreover, soybean has experienced a whole-genome duplication after diverging from common bean. Interestingly, our results slightly deviated from the earlier reports in common bean for all the three contexts (Kim et al., 2015). The deviation in the methylation ratios in this study may be attributed to the genotype-specific DNA methylation variation (Meso-American vs Andean), differences in leaf stages (primary vs. trifoliate) and collection time-points (14-day-old vs. 18-day-old) used while analyzing the bean methylome.

\section{Promoter and Gene-Body Methylation}

Promoter methylation and genic methylation have been implicated in diverse regulatory roles. Generally, cytosine methylation in promoter regions results in transcriptional repression in plants. A single methylated cytosine in a promoter region can significantly affect the expression level of the corresponding gene. About $16.6 \%$ of methylated single CGs identified in the promoter adjacent to transcriptional start sites decreased gene expression in humans (Medvedeva et al., 2014). CG and CHG promoter methylation typically causes repression of adjacent genes, as reported in Arabidopsis, maize, and soybean (Zhang et al., 2006; Song et al., 2013; West et al., 2014). The role of gene-body methylation in controlling alternative promoters and gene splicing has been implicated in mammals (Lou et al., 2014). Gene-body methylation is considered as an evolutionary consequence and it is conserved among plant orthologs (Takuno and Gaut, 2012). With this background we attempted to understand the promoter and genic methylation in common bean.

After annotating the methylated sites for genomic features, we identified higher CG methylation levels in both promoter and genic regions than $\mathrm{CHG}$ and $\mathrm{CHH}$ contexts (Figure 3A). Moreover, we found relatively higher CG methylation levels in genes than in promoters. Conversely, the $\mathrm{CHG}$ and $\mathrm{CHH}$ methylation levels were highest in promoters than in genes, which is consistent with the findings in Arabidopsis (Cokus et al., 2008). We further confirmed that the CG methylation levels are higher in the annotated genomic features (Figure 3B). The most significant MeDIP-Seq peaks were found to correlate with high levels of both CG and CHG methylation (Table 3). A study in Arabidopsis revealed that important functional genes evolved slowly when they contained methylation in the genebody (Takuno and Gaut, 2012). Another study in Arabidopsis found high levels of methylation in gene-bodies (Zhang et al., 2006). Further, it was found that single-copy genes in soybean and common bean were methylated more frequently than duplicated genes (Kim et al., 2015). This suggests that gene-body methylation may have an important evolutionary role, possibly in protecting genes from mutation. DNA methylation has been implicated in physiological and developmental processes in plants. A study conducted in cotton suggested the role of $\mathrm{CHH}$ methylation in time-of-day and time-of-year memory (Jin et al., 2013). The fluctuation in DNA methylation pattern in response to different time intervals (Jin et al., 2013) and DNA methylation diversity between genotypes have been proposed in cotton (Osabe et al., 2014). Similarly, we presume differences in DNA methylation found between G19833 (Kim et al., 2015) and Sierra (current study) in common bean is partially due to the fact that two genotypes are derived from different gene pools.

\section{Comparison of BS-Seq and MeDIP-Seq}

As BS-Seq and MeDIP-Seq employ different mechanisms for determining methylated cytosines, the bioinformatic output of each method is also different. In the current study, BS-Seq ( $\sim 214$ million reads) generated approximately 10 -fold more reads than that of the immunoprecipitated reads via MeDIP-Seq ( 22.5 million reads). The cost per sample was about a twofold difference, BS-Seq being the more expensive option. Due to the expense, targeted bisulfite conversion, and sequencing is often chosen for specific genes or regions. However, this option gives much less information than both high-throughput sequencing technologies MeDIP-Seq and BS-Seq.

Since MeDIP-seq is affinity-based and the output is derived from peak shapes, the context of the methylated cytosine is more complicated to determine and less informative. BS-Seq yields individual nucleotide output, therefore, the specific context that is methylated is much more obvious. Because of this difference, precise trends about methylation in specific contexts cannot be made from MeDIP-Seq data. Methylation trends in annotated regions can be examined; for example Table 2 shows the total 
A

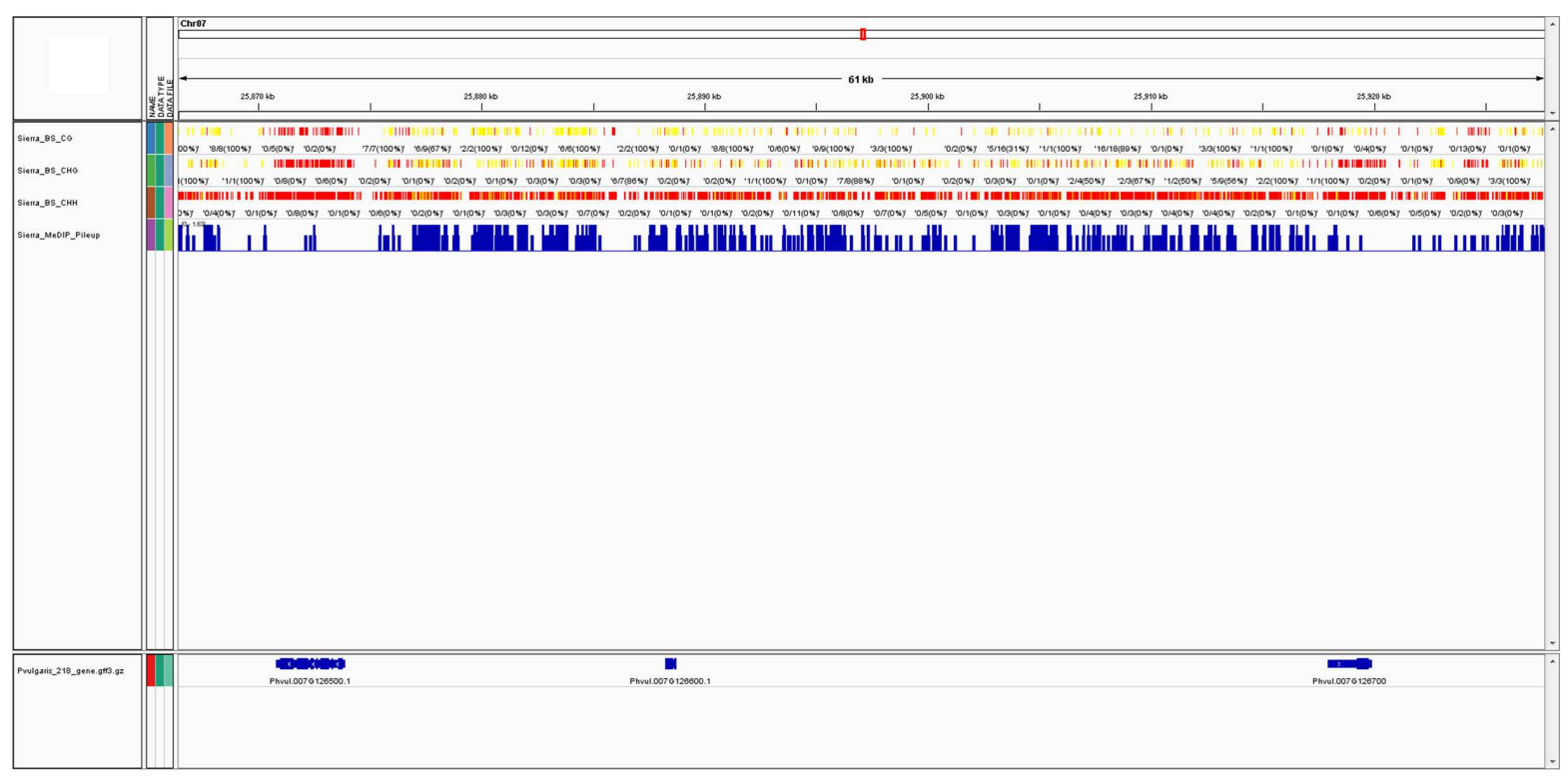

B

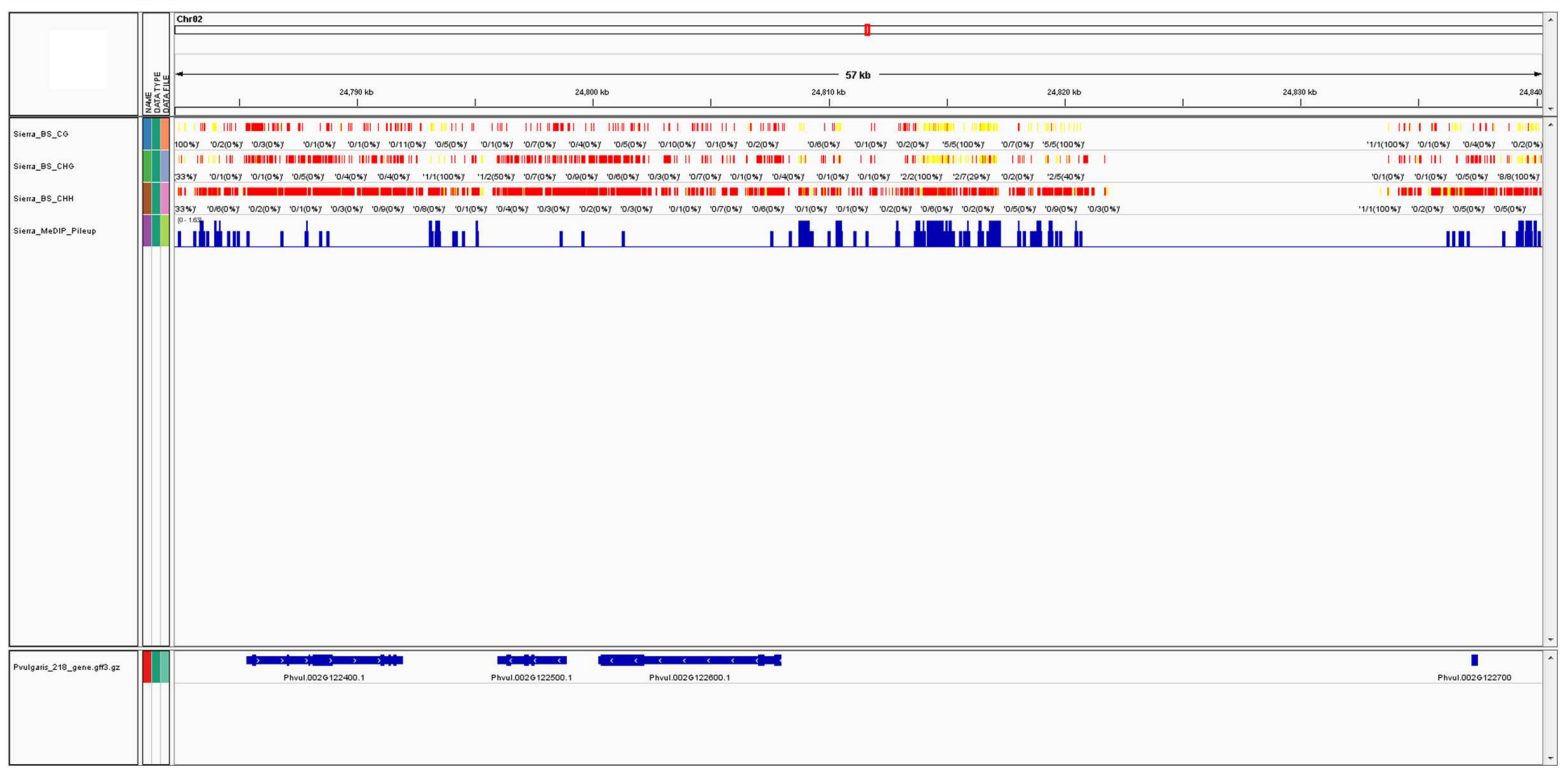

FIGURE 4 | (A) MeDIP-Seq and BS-Seq data can be visualized in a single window with a genome viewer. In the screenshot, BS-Seq tracks are included for a segment of chromosome 7 (Chr07, position 25,866,407-25,927,674) showing CG, CHG, and CHH sites for from BS-Seq data, and the fourth track is of MeDIP-Seq peaks. These tracks were viewed via the Integrative Genome Viewer (IGV) program. Chr07 is one of the most highly methylated bean chromosomes, as determined by MeDIP-Seq peak density. Yellow indicates high methylation, while red indicates low or no methylation. (B) Screenshot of a segment of chromosome 2 (Chr02, position 24,782,253-24,840,303) via IGV, which is one of the least methylated bean chromosomes, as determined by MeDIP-Seq peak density. Both figures show approximately $60 \mathrm{~Kb}$ of sequence at the furthest possible zoom scale to visualize BS-Seq and MeDIP-Seq data in one window.

peaks, promoter, and genic peaks per chromosome. Genome viewers can be used to compare multiple datasets aligned to the same reference genome, we compared BS-Seq and MeDIP-Seq (Figures 4A,B), but other datasets such as RNA-Seq and ChIPSeq could also be viewed simultaneously (Thorvaldsdottir et al., 2013).

Since researchers are often interested in finding genomewide differences between a control and treatment, the use of either method would be useful. If only large changes in DNA methylation patterns are of interest, MeDIP-Seq may be the better option, since it is less expensive and uses an affinitybased approach. However, if determining small changes or very specific changes in DNA methylation are desired, BS-Seq is the better option, as it provides much more data. Further, the use of targeted BS-Seq would be the very least expensive per sample. With decreasing sequencing costs, in the future BS-Seq will likely be the best option, as long as there are means to store the data. 


\section{CONCLUSION}

In summary, this is the first genome-wide DNA methylation profiling study in the Meso-American common bean cultivar Sierra using NGS approaches such as BS-Seq and MeDIP-Seq. Methylation statuses of each of the three DNA methylation contexts CG $(\sim 50 \%), \mathrm{CHG}(\sim 30)$, and $\mathrm{CHH}$ $(\sim 2.7 \%)$ were determined from BS-Seq. Overall promoter and genic methylation trends were established. CG was the most commonly methylated context, and was more common in promoters than in genes. The opposite trend was found in $\mathrm{CHG}$ and $\mathrm{CHH}$ methylation contexts. The majority (93.2\%) of MeDIP-Seq peaks were found in intragenic regions.

\section{MATERIALS AND METHODS}

\section{Plant Material}

The common bean (P. vulgaris) cultivar "Sierra" a pinto bean derived from the Meso-American gene pool was used in this study. Seeds were germinated for 2-3 days, on wet filter paper in petridishes and then planted in $6^{\prime \prime}$ pots, filled with Promix $\mathrm{Bx}$ mycorrhizae soil, and grown in a greenhouse, at Delaware State University, Dover, DE, USA. Three separate times, three plants each were grown in the greenhouse under standard conditions, with a photoperiod of approximately $14 \mathrm{~h}$ days $/ 10 \mathrm{~h}$ nights at $28 / 20^{\circ} \mathrm{C}$ (Ayyappan et al., 2015). At 14 days after planting, leaves from the three plants were harvested separately, high quality DNA was extracted, and equal amounts of DNA were combined to represent a single sample from these three plants, called Pool 1. Similarly, the second set of three plants, and the third set of three plants were processed to obtain Pool 1, Pool 2, and Pool 3. Finally, equal amounts of DNA from pools 1, 2, and 3 were combined for the BS-seq experiment. The same sample that was used for BS-seq was also used for the MeDIP-seq experiment so as to help us obtain a direct comparison of these two methods and their efficiency in identifying methylated regions in the genome. Leaves from each plant were collected after two weeks, flash frozen in liquid nitrogen, and stored in a $-80^{\circ} \mathrm{C}$ freezer until DNA was extracted.

\section{DNA Extraction}

DNA was extracted from 2-week old leaves using a CTABbased protocol as previously described (Doyle, 1990). The quantity and quality of DNA were determined by $1.0 \%$ agarose gel electrophoresis and Nanodrop 2000 spectrophotometer (Thermo Scientific, Wilmington, DE, USA). The experiment included a single pooled DNA sample collected from the leaves of nine different plants (biological replicates) and utilized the same sample for generating the MeDIP-Seq and BSSeq libraries to maintain uniform experimental conditions. Both the libraries were sequenced on the Illumina/HiSeq-2500 platform.

\section{Methylated Cytosine Sequencing Methyl-MaxiSeq ${ }^{\mathrm{TM}}$ Library Construction}

Methyl-MaxiSeq ${ }^{\mathrm{TM}}$ EpiQuest libraries were prepared from $100 \mathrm{ng}$ of genomic DNA, which was bisulfite-treated using Zymo Research EZ DNA Methylation - Lightning ${ }^{\text {TM }}$ kit (Cat\#: D5030, Zymo Research, Irvine, CA, USA). The bisulfite conversion rate was determined to be $>99 \%$. The bisulfite-converted DNA was subjected to a series of amplifications, which include a primer that contained part of the adapter sequence and four random nucleotides, followed by adding the remaining adapter sequence and barcoding the fragments, respectively. All PCR products were purified using the DNA Clean \& Concentrator-5 ${ }^{\mathrm{TM}}$ (Cat\#: D4003, Zymo Research, Irvine, CA, USA). Library fragment size and concentration was checked using the Agilent 2200 TapeStation instrument and sequenced on the Illumina HiSeq 2500 platform (Illumina Inc., San Diego, CA, USA).

\section{Methyl-MaxiSeq $^{\mathrm{TM}}$ Sequence Alignments and Data Analysis}

Sequence reads from bisulfite-treated EpiQuest libraries were identified using standard Illumina base-calling software and then analyzed using a Zymo Research proprietary analysis pipeline written in Python and using Bismark (http://www.bio informatics.babraham.ac.uk/projects/bismark/) as the alignment software for analysis (Krueger and Andrews, 2011). Index files were constructed by bismark_genome_preparation command using the entire reference genome. Non_directional and all other default parameters were applied while running Bismark. The methylation level of each sampled cytosine was estimated as the number of reads reporting a $\mathrm{C}$, divided by the total number of reads reporting a $\mathrm{C}$ or $\mathrm{T}$. Promoter and gene body annotations were added using $P$. vulgaris genome annotations available at Phytozome (V1.0).

\section{MeDIP-Seq Library Construction}

Libraries for MeDIP-Seq were prepared following immunoprecipitation using the Zymo Research DNA Methylation IP Kit (Cat \#D5101, Zymo Research, Irvine, CA, USA). Immunoprecipitated DNA was subjected to a series of amplifications as described above for preparing bisulfiteconverted libraries. All PCR products were purified using the Zymo Research DNA Clean \& Concentrator-5 ${ }^{\text {TM }}$ (Cat\#: D4003, Zymo Research, Irvine, CA, USA). The input DNA library was prepared from pooled sample DNA that was fragmented and denatured. Libraries were quantified using the Agilent 2200 TapeStation and by qPCR. Sample concentrations were normalized to $4 \mathrm{nM}$, and then sequenced on the Illumina HiSeq 2500 platform (Illumina Inc., San Diego, CA, USA).

\section{MeDIP-Seq Sequence Alignments and Data Analysis}

Sequencing reads were aligned to the reference genome (V1.0) by Bowtie using best mode and other default parameters. Peak calling was done by "MACS2 callpeak" using input DNA as a control. BIGWIG files were generated from the coverage for visualization purposes (Zhang et al., 2008). Sequences derived 
from MeDIP-seq BS-seq libraries were submitted to the short read archives at NCBI BioProject\#PRJNA306503.

\section{AUTHOR CONTRIBUTIONS}

MC conceived and performed experiments, interpreted the data, and developed the seminal draft of the manuscript. VS performed bioinformatic analysis, interpreted the data, and contributed significantly in drafting the manuscript. $\mathrm{KH}$ supervised and contributed to the drafting of the manuscript. VK conceived, supervised the whole project, and contributed to the drafting and editing of the manuscript.

\section{FUNDING}

We sincerely acknowledge funding from the National Science Foundation EPSCoR Grant No. IIA-1301765 and the State of Delaware (VK).

\section{REFERENCES}

Akond, G. M., Crawford, H., Berthold, J., Taulkder, Z. I., and Hossain, K. (2011). Minerals $\mathrm{Zn}, \mathrm{Fe}, \mathrm{Ca}$, and $\mathrm{Mg}$ and antinutirent phytic acid constitutents in common bean. Am. J. Food Technol. 6, 235-243. doi: 10.3923/ajft.2011. 235.243

Albert, V. A., Barbazuk, W. B., DePamphilis, C. W., Der, J. P., LeebensMack, J., Ma, H., et al. (2013). The amborella genome and the evolution of flowering plants. Science 342, 1241089-1241089. doi: 10.1126/science.12 41089

Ayyappan, V., Kalavacharla, V., Thimmapuram, J., Bhide, K. P., Sripathi, V. R., Smolinski, T. G., et al. (2015). Genome-wide profiling of histone modifications (H3K9me2 and $\mathrm{H} 4 \mathrm{~K} 12 \mathrm{ac}$ ) and gene expression in rust (Uromyces appendiculatus) inoculated common bean (Phaseolus vulgaris L.). PLoS ONE 10:e0132176. doi: 10.1371/journal.pone.0132176

Beebe, S. E., Rao, I. M., Blair, M. W., and Acosta-Gallegos, J. A. (2013). Phenotyping common beans for adaptation to drought. Front. Physiol. 4:35. doi: 10.3389/fphys.2013.00035

Bitocchi, E., and Nanni, L. (2012). Mesoamerican origin of the common bean (Phaseolus vulgaris L.) is revealed by sequence data. Proc. Natl. Acad. Sci. U.S.A. $109,788-796$.

Blair, M. W. (2013). Mineral biofortification strategies for food staples: the example of common bean. J. Agric. Food Chem. 61, 8287-8294. doi: 10.1021/jf $400774 y$

Boyko, A., Kathiria, P., Zemp, F. J., Yao, Y., Pogribny, I., and Kovalchuk, I. (2007). Transgenerational changes in the genome stability and methylation in pathogen-infected plants: (Virus-induced plant genome instability). Nucleic Acids Res. 35, 1714-1725. doi: 10.1093/nar/gkm029

Brinkman, A. B., Gu, H., Bartels, S. J. J., Zhang, Y., Matarese, F., Simmer, F., et al. (2012). Sequential ChIP-bisulfite sequencing enables direct genome-scale investigation of chromatin and DNA methylation cross-talk. Genome Res. 22, 1128-1138. doi: 10.1101/gr.133728.111

Câmara, C., Urrea, C., and Schlegel, V. (2013). Pinto beans (Phaseolus vulgaris L.) as a functional food: Implications on human health. Agriculture 3, 90-111. doi: 10.3390/agriculture3010090

Cao, X., Aufsatz, W., Zilberman, D., Mette, M. F., Huang, M. S., Matzke, M., et al. (2003). Role of the DRM and CMT3 methyltransferases in RNAdirected DNA methylation. Curr. Biol. 13, 2212-2217. doi: 10.1016/j.cub.2003. 11.052

Chodavarapu, R. K., Feng, S., Ding, B., Simon, S. A., Lopez, D., Jia, Y., et al. (2012). Transcriptome and methylome interactions in rice hybrids. Proc. Natl. Acad. Sci. U.S.A. 109, 12040-12045. doi: 10.1073/pnas.1209297109

\section{ACKNOWLEDGMENT}

The authors acknowledge the assistance of Rita K. Hayford and other members of the Molecular Genetics and Epigenomics Laboratory at Delaware State University in editing and preparing this manuscript.

\section{SUPPLEMENTARY MATERIAL}

The Supplementary Material for this article can be found online at: http://journal.frontiersin.org/article/10.3389/fpls.2016. 00447

FIGURE S1 | The peak shapes for the input and immunoprecipitated DNA samples are shown.

FIGURE S2 | Read coverage across the genome for each methylation context is shown. The top is $\mathrm{CG}$, middle is $\mathrm{CHG}$, and bottom is $\mathrm{CHH}$ contexts. Coverage read ranges from 0 (no coverage), to 1-4 reads, 5-10 reads, 11-20 reads, and 20 or more reads.

Cokus, S. J., Feng, S., Zhang, X., Chen, Z., Merriman, B., Haudenschild, C. D., et al. (2008). Shotgun bisulphite sequencing of the Arabidopsis genome reveals DNA methylation patterning. Nature 452, 215-219. doi: 10.1038/nature 06745

Doyle, J. J. (1990). Isolation of plant DNA from fresh tissue. Focus 12, 13-15.

Eichten, S. R., Briskine, R., Song, J., Li, Q., Swanson-Wagner, R., Hermanson, P. J., et al. (2013). Epigenetic and genetic influences on DNA methylation variation in maize populations. Plant Cell 25, 2783-2797. doi: 10.1105/tpc.113. 114793

Feng, S., Cokus, S. J., Zhang, X., Chen, P.-Y., Bostick, M., Goll, M. G., et al. (2010). Conservation and divergence of methylation patterning in plants and animals. Proc. Natl. Acad. Sci. U.S.A. 107, 8689-8694. doi: 10.1073/pnas.1002720107

Gent, J. I., Ellis, N. A., Guo, L., Harkess, A. E., Yao, Y., Zhang, X., et al. (2013). CHH islands: de novo DNA methylation in near-gene chromatin regulation in maize. Genome Res. 23, 628-637. doi: 10.1101/gr.146985.112.as

Gepts, P., Aragão, F. J. L., Barros, E., De Blair, M. W., Brondani, R., Broughton, W. et al. (2008). Genomics of Phaseolus beans, a major source of dietary protein and micronutrients in the tropics. Genomics 1, 113-143. doi: 10.1007/978-0387-71219-2_5

Greaves, I. K., Groszmann, M., Ying, H., Taylor, J. M., Peacock, W. J., and Dennis, E. S. (2012). Trans chromosomal methylation in Arabidopsis hybrids. Proc. Natl. Acad. Sci. U.S.A. 109, 3570-3575. doi: 10.1073/pnas.1201043109

Henderson, I. R., Chan, S. R., Cao, X., Johnson, L., and Jacobsen, S. E. (2010). Accurate sodium bisulfite sequencing in plants. Epigenetics 5, 47-49. doi: 10.4161/epi.5.1.10560

Hseih, T., Ibarra, C. A., Silva, P., Zemach, A., Eshed-Williams, L., Fischer, R. L., et al. (2009). Genome-wide demethylation of Arabidopsis endosperm. Science 324, 1451-1454. doi: 10.1126/science.1172417

Hughes, T., Webb, R., Fei, Y., Wren, J. D., and Sawalha, A. H. (2010). DNA methylome in human $\mathrm{CD}^{+}{ }^{+} \mathrm{T}$ cells identifies transcriptionally repressive and non-repressive methylation peaks. Genes Immun. 11, 554-560. doi: 10.1038/gene.2010.24

Jin, X., Pang, Y., Jia, F., Xiao, G., Li, Q., and Zhu, Y. (2013). A potential role for CHH DNA methylation in cotton fiber growth patterns. PLoS ONE 8:e60547. doi: 10.1371/journal.pone.0060547

Kim, K. D., El Baidouri, M., Abernathy, B., Iwata-Otsubo, A., Chavarro, C., Gonzales, M., et al. (2015). A comparative epigenomic analysis of polyploidyderived genes in soybean and common bean. Plant Physiol. 168, 1433-1447. doi: 10.1104/pp.15.00408

Kim, K. D., El Baidouri, M., and Jackson, S. A. (2014). Accessing epigenetic variation in the plant methylome. Brief. Funct. Genomics 13, 318-327. doi: 10.1093/bfgp/elu003 
Krueger, F., and Andrews, S. R. (2011). Bismark: a flexible aligner and methylation caller for Bisulfite-Seq applications. Bioinformatics 27, 1571-1572. doi: 10.1093/bioinformatics/btr167

Laird, P. W. (2010). Principles and challenges of genome-wide DNA methylation analysis. Nat. Rev. Genet. 11:191. doi: 10.1038/nrg2732

Law, J. A., and Jacobsen, S. E. (2010). Establishing, maintaining and modifying DNA methylation patterns in plants and animals. Nat. Rev. Genet. 11, 204-220. doi: $10.1038 / \operatorname{nrg} 2719$

Li, X., Wang, X., He, K., Ma, Y., Su, N., He, H., et al. (2008). High-resolution mapping of epigenetic modifications of the rice genome uncovers interplay between DNA methylation, histone methylation, and gene expression. Plant Cell 20, 259-276. doi: 10.1105/tpc.107.056879

Li, X., Zhu, J., Hu, F., Ge, S., Ye, M., Xiang, H., et al. (2012). Single-base resolution maps of cultivated and wild rice methylomes and regulatory roles of DNA methylation in plant gene expression. BMC Genomics 13:300. doi: 10.1186/1471-2164-13-300

Lister, R., and Ecker, J. R. (2009). Finding the fifth base: genome-wide sequencing of cytosine methylation. Genome Res. 19, 959-966. doi: 10.1101/gr.083451.108

Lister, R., O’Malley, R. C., Tonti-Filippini, J., Gregory, B. D., Berry, C. C., Millar, A. H., et al. (2009). Highly integrated single-base resolution maps of the epigenome in Arabidopsis. Cell 133, 523-536. doi: 10.1016/j.cell.2008.03.029

Lou, S., Lee, H.-M., Qin, H., Li, J.-W., Gao, Z., Liu, X., et al. (2014). Whole-genome bisulfite sequencing of multiple individuals reveals complementary roles of promoter and gene body methylation in transcriptional regulation. Genome Biol. 15:408. doi: 10.1186/PREACCEPT-1031081530108509

Madlung, A., and Comai, L. (2004). The effect of stress on genome regulation and structure. Ann. Bot. 94, 481-495. doi: 10.1093/aob/mch172

Medvedeva, Y. A., Khamis, A. M., Kulakovskiy, I. V., Ba-Alawi, W., Bhuyan, M. S. I., Kawaji, H., et al. (2014). Effects of cytosine methylation on transcription factor binding sites. BMC Genomics 15:119. doi: 10.1186/1471-2164-15-119

Mitchell, D. C., Lawrence, F. R., Hartman, T. J., and Curran, J. M. (2009). Consumption of dry beans, peas, and lentils could improve diet quality in the US population. J. Am. Diet. Assoc. 109, 909-913. doi: 10.1016/j.jada.2009. 02.029

Miura, A., Nakamura, M., Inagaki, S., Kobayashi, A., Saze, H., and Kakutani, T. (2009). An Arabidopsis jmjC domain protein protects transcribed genes from DNA methylation at CHG sites. EMBO J. 28, 1078-1086. doi: 10.1038/emboj.2009.59

Osabe, K., Clement, J. D., Bedon, F., Pettolino, F. A., Ziolkowski, L., Llewellyn, D. J., et al. (2014). Genetic and DNA methylation changes in cotton (Gossypium) genotypes and tissues. PLoS ONE 9:e86049. doi: 10.1371/journal.pone.0086049

Petry, N., Boy, E., Wirth, J., and Hurrell, R. (2015). Review: The potential of the common bean (Phaseolus vulgaris) as a vehicle for iron biofortification. Nutrients 7, 1144-1173. doi: 10.3390/nu7021144

Regulski, M., Lu, Z., Kendall, J., Donoghue, M. T. A., Reinders, J., Llaca, V., et al. (2013). The maize methylome influences mRNA splice sites and reveals widespread paramutation-like switches guided by small RNA. Genome Res. 23, 1651-1662. doi: 10.1101/gr.153510.112

Saze, H., Tsugane, K., Kanno, T., and Nishimura, T. (2012). DNA methylation in plants: relationship to small RNAs and histone modifications, and functions in transposon inactivation. Plant Cell Physiol. 53, 766-784. doi: $10.1093 / \mathrm{pcp} / \mathrm{pcs} 008$

Schmitz, R. J., He, Y., Valdés-lópez, O., Res, G., Gent, J. I., Ellis, N. A., et al. (2013). Epigenome-wide inheritance of cytosine methylation variants in a recombinant inbred population. Genome Res. 23, 1663-1674. doi: 10.1101/gr.152538.112

Schmutz, J., McClean, P. E., Mamidi, S., Wu, G. A., Cannon, S. B., Grimwood, J., et al. (2014). A reference genome for common bean and genome-wide analysis of dual domestications. Nat. Genet. 46, 707-713. doi: 10.1038/ng.3008

Seymour, D. K., Koenig, D., Hagmann, J., Becker, C., and Weigel, D. (2014). Evolution of DNA methylation patterns in the Brassicaceae is driven by differences in genome organization. PLoS Genet. 10:e1004785. doi: 10.1371/journal.pgen.1004785

Shen, H., He, H., Li, J., Chen, W., Wang, X., Guo, L., et al. (2012). Genomewide analysis of DNA methylation and gene expression changes in two Arabidopsis ecotypes and their reciprocal hybrids. Plant Cell 24, 875-892. doi: 10.1105/tpc. 111.094870
Song, Q.-X., Lu, X., Li, Q.-T., Chen, H., Hu, X.-Y., Ma, B., et al. (2013). Genomewide analysis of DNA methylation in soybean. Mol. Plant 6, 1961-1974. doi: $10.1093 / \mathrm{mp} / \mathrm{sst} 123$

Statham, A. L., Robinson, M. D., Song, J. Z., Coolen, M. W., Stirzaker, C., and Clark, S. J. (2012). Bisulfite sequencing of chromatin immunoprecipitated DNA (BisChIP-seq) directly informs methylation status of histone-modified DNA. Genome Res. 22, 1120-1127. doi: 10.1101/gr.132076.111

Stevens, M., Cheng, J. B., Li, D., Xie, M., Hong, C., Maire, C. L., et al. (2013). Estimating absolute methylation levels at single-CpG resolution from methylation enrichment and restriction enzyme sequencing methods. Genome Res. 23, 1541-1553. doi: 10.1101/gr.152231.112

Stroud, H., Ding, B., Simon, S. A., Feng, S., Bellizzi, M., Pellegrini, M., et al. (2013a). Plants regenerated from tissue culture contain stable epigenome changes in rice. Elife 2, 1-14. doi: 10.7554/eLife.00354

Stroud, H., Do, T., Du, J., Zhong, X., Feng, S., Johnson, L., et al. (2013b). Non-CG methylation patterns shape the epigenetic landscape in Arabidopsis. Nat. Struct. Mol. Biol. 21, 64-72. doi: 10.1038/nsmb.2735

Taiwo, O., Wilson, G. A., Morris, T., Seisenberger, S., Reik, W., Pearce, D., et al. (2012). Methylome analysis using MeDIP-seq with low DNA concentrations. Nat. Protoc. 7, 617-636. doi: 10.1038/nprot.2012.012

Takuno, S., and Gaut, B. S. (2012). Body-methylated genes in Arabidopsis thaliana are functionally important and evolve slowly. Mol. Biol. Evol. 29, 219-227. doi: $10.1093 / \mathrm{molbev} / \mathrm{msr} 188$

Taylor, K. H., Kramer, R. S., Davis, J. W., Guo, J., Duff, D. J., Xu, D., et al. (2007). Ultradeep bisulfite sequencing analysis of DNA methylation patterns in multiple gene promoters by 454 sequencing. Cancer Res 67, 8511-8518. doi: 10.1158/0008-5472.CAN-07-1016

Thorvaldsdottir, H., Robinson, J. T., and Mesirov, J. P. (2013). Integrative genomics viewer (IGV): high-performance genomics data visualization and exploration. Brief. Bioinform. 14, 178-192. doi: 10.1093/bib/bbs017

Verhoeven, K. J. F., Jansen, J. J., van Dijk, P. J., and Biere, A. (2010). Stress-induced DNA methylation changes and their heritability in asexual dandelions. New Phytol. 185, 1108-1118. doi: 10.1111/j.1469-8137.2009.03121.x

Wang, J., Marowsky, N. C., and Fan, C. (2014). Divergence of gene body DNA methylation and evolution of plant duplicate genes. PLOS ONE 9:e110357. doi: 10.1371/journal.pone.0110357

West, P. T., Li, Q., Ji, L., Eichten, S. R., Song, J., Vaughn, M. W., et al. (2014). Genomic distribution of $\mathrm{H} 3 \mathrm{~K} 9 \mathrm{me} 2$ and DNA methylation in a maize genome. PLoS ONE 9:e105267. doi: 10.1371/journal.pone.0105267

Zemach, A., Kim, M. Y., Silva, P., Rodrigues, J. A., Dotson, B., Brooks, M. D., et al. (2010). Local DNA hypomethylation activates genes in rice endosperm. Proc. Natl. Acad. Sci. U.S.A. 107, 18729-18734. doi: 10.1073/pnas.1009 695107

Zhang, X., Yazaki, J., Sundaresan, A., Cokus, S., Chan, S. W.-L., Chen, H., et al. (2006). Genome-wide high-resolution mapping and functional analysis of DNA methylation in Arabidopsis. Cell 126, 1189-1201. doi: 10.1016/j.cell.2006. 08.003

Zhang, Y., Liu, T., Meyer, C. A., Eeckhoute, J., Johnson, D. S., Bernstein, B. E., et al. (2008). Model-based Analysis of ChIP-Seq (MACS). Genome Biol. 9:R137. doi: 10.1186/gb-2008-9-9-r137

Conflict of Interest Statement: The authors declare that the research was conducted in the absence of any commercial or financial relationships that could be construed as a potential conflict of interest.

The reviewer JR and handling Editor declared their shared affiliation, and the handling Editor states that the process nevertheless met the standards of a fair and objective review.

Copyright (c) 2016 Crampton, Sripathi, Hossain and Kalavacharla. This is an openaccess article distributed under the terms of the Creative Commons Attribution License (CC BY). The use, distribution or reproduction in other forums is permitted, provided the original author(s) or licensor are credited and that the original publication in this journal is cited, in accordance with accepted academic practice. No use, distribution or reproduction is permitted which does not comply with these terms. 